\title{
Automated Segmentation of Retinal Blood Vessels using Optimized Gabor Filter with Local Entropy Thresholding
}

\author{
Saumitra Kumar Kuri \\ Department of Applied Physics, Electronics \& \\ Communication Engineering \\ University of Chittagong \\ Chittagong, Bangladesh
}

\author{
Jayant V. Kulkarni \\ Department of Instrumentation \& Control \\ Engineering \\ Vishwakarma Institute of Technology \\ Pune, India
}

\begin{abstract}
Blood vessel in retinal image plays a vital role in medical diagnosis of many diseases. Diabetic retinopathy is one of the diseases which damages the retina and leads to blindness. Segmentation of blood vessels is helpful for ophthalmologists and this paper presents a new automatic method to extract blood vessels with high accuracy. This algorithm is comprised of optimized Gabor filter with local entropy thresholding for vessels segmentation under various normal or abnormal conditions. The frequency and orientation of Gabor filter are tuned to match that of a part of blood vessels to be enhanced in a green channel image. Segmentation of blood vessels pixels are classified by local entropy thresholding technique in this method. The performance of the proposed algorithm is evaluated by MATLAB software with DRIVE database.
\end{abstract}

\section{General Terms}

Bio-informatics, Computer Aided Diagnosis system

\section{Keywords}

Retinal image, Blood vessels, Diabetic retinopathy, Optimized Gabor filter, Local entropy thresholding.

\section{INTRODUCTION}

Diabetic retinopathy (DR) is the result of damage caused by diabetes to the small blood vessels located in the retina. Blood vessels damaged from diabetic retinopathy can cause vision loss. Diabetic retinopathy is a leading cause of adult blindness, and screening can reduce the incidence. Screening just increases the chances that a condition will be avoided, found early, or are able to be cured. It is widely recommended that all persons with diabetes mellitus should be regularly screened for diabetic retinopathy [1]

Computer based analysis for automated segmentation of blood vessels in retinal images will help ophthalmologists screen larger populations for vessel abnormalities. A wide variety of approaches have been proposed for retina blood vessels segmentation. Many image processing methods proposed for retinal vessels extraction [4] [5] [6] [7] [8] [9] [10]. This literature is based on optimized Gabor filter with local entropy thresholding. Gabor filters have been widely applied to image processing and computer vision application problems such as face recognition and texture segmentation [8]. Optimized Gabor filter methods often produce false positive detections and fail to detect vessel of different widths. Also detection process much more complicated when retinal image abnormal condition.

This paper has been proposed a much robust and fast method of retinal blood vessels extraction using optimized Gabor filter with local entropy thresholding.

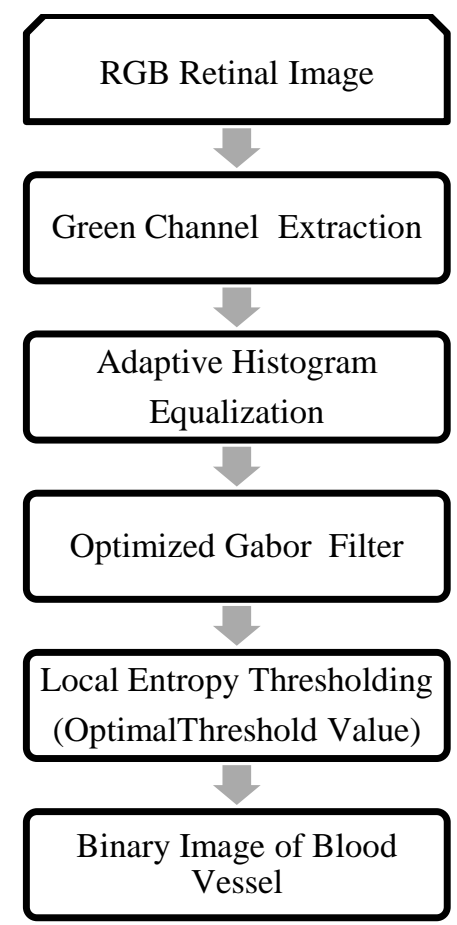

Fig 1: Flow chart of the proposed methodology for blood vessel segmentation in retinal image

\section{MATERIALS AND METHODS}

DRIVE database [30] is used for this analysis. Every image was capture at $584 \times 565$ pixels, 8 bits per color in TIFF format.

Blood vessels usually have poor local contrast compare to background. The proposed method uses the following steps: (i) Green channel extraction, (ii) Adaptive Histogram Equalization, (iii) Optimized Gabor filter, (iv) Local Entropy Thersholding (v) Binary conversion. The green channel is inverted before the application of the Gabor Filter transform to it, so that the vessels appear brighter than the background. Fig.1. shows the overall procedure of the proposed method.

\subsection{Preprocessing}

Preprocessing is applied to eliminate the noises in the fundus image. Regarding the acquisition process, retinal images have often low contrast that cause to hardly detect the blood vessels. This method is to improve the image dynamic range to prepare images for next step, detection the blood vessels, and attain to higher accuracy and precision of segmentation. Concerning our purpose, contrast enhancement, the green channel of colored retinal images is used, because compare to other channels it has the highest contrast [4]. Combining advantages of brightness in red channel decreasing the contrast between the abnormalities and the retinal 
background; this helps to reduce some responses from abnormalities which do not resemble any blood vessels that would otherwise decrease the performance of blood vessels segmentation methods. Contrast-limited adaptive histogram equalization is used for this analysis that enhancing the contrast of the green channel retinal image.

\subsection{Optimized Gabor Filter}

Gabor filters have been widely used for multi-directional analysis in image processing. In this algorithm optimized Gabor filter is used for detecting the blood vessel in retinal image. The optimized Gabor Filters are a set of orientation and frequency sensitive band pass filters which have the optimal localization in both the frequency contents of the patterns [8]. The optimized Gabor filter kernels are sinusoids modulated.

$$
\begin{aligned}
& \sigma_{x}=k \\
& \sigma_{y}=\frac{\sigma_{x}}{\gamma} \\
& x_{\theta}=x \cos \theta+y \sin \theta \\
& y_{\theta}=-x \sin \theta+y \cos \theta
\end{aligned}
$$

Optimized Gabor filter kernel:

$$
g_{\theta}(x, y)=\exp \left\{-\frac{1}{2}\left(\frac{x_{\theta}^{2}}{\sigma_{x}}+\frac{\left(\gamma y_{\theta}\right)^{2}}{\sigma_{y}}\right)\right\} \cos \left(2 \pi \frac{x_{\theta}}{\lambda}+\psi\right)
$$

Where,

$\sigma_{x}$ : Standard deviation of Gaussian in $\mathrm{x}$ direction along the filter that determine the bandwidth of the filter.

$\sigma_{y}$ : Standard deviation of Gaussian filter that control the orientation selectivity of the filter.

$\theta$ : Orientation of the filter, an angle of zero gives a filter responds to vertical feature.

$\lambda$ : Wavelength of the cosine factor of the Gabor filter kernel i.e. preferred wavelength of this filter.

$\gamma$ : Spatial aspect ratio, specifies the ellipticity of the support of the Gabor function

\section{$\psi:$ Phase offset}

The optimization Gabor filter kernel $(9 \times 7$ matrix) is rotated in different rotations with the optimized parameters set as follows:

$\sigma_{x} \in[3.91,4], \lambda \in[5.1,5.3], \gamma \in[1.2,1.4]$

$\sigma_{x}=3.91$

$\lambda=5.1$

$\gamma=1.3$

$\psi=2 \pi$

$\sigma_{x}$ is required so that the shapes of the filter are invariant to the scale. The width of the vessels is found to lie within a range of 2-14 pixels $(40-200 \mu \mathrm{m})$. Here, $\lambda$ and $\gamma$ values maintain false positive rate. $\psi$ always $(2 \pi)$ rotation phase in this method. The optimized parameters are to be derived by taking into account of size of the lines structures to be detected. Only six optimized Gabor filters with different orientations ( 0 to $360^{\circ}$ intervals of sixty degrees) are used to convolve with the preprocessing image. The magnitude of each response is retained and combined to generate the result image.
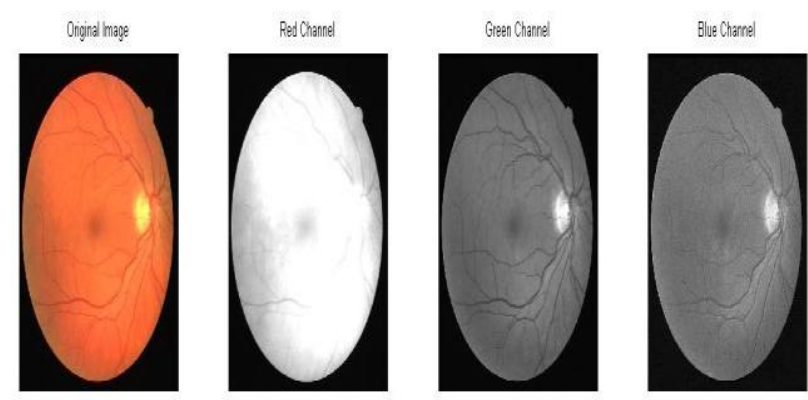

Fig 2: Original image with extraction channels

\subsection{Local Entropy Thresholding}

An image can be viewed as an information source with a probability vector described by its grey-level image histogram, the entropy of the histogram can be used to represent a certain level of information contained in the image. Pun [16] and Kapur et al. [17] had taken this concept to derive entropy thresholding methods. However, their approaches did not take into account the correlation among grey levels. As a result, two different images with an identical image histogram will result in the same threshold value [18]. One way to resolve this problem is to consider the grey-level co-occurrence matrix, which contains the information of greylevel transitions in an image. In the proposed method the grey-level co-occurrence matrix developed by Haralick et al [19] is used to derive the Haralick texture feature for retinal image segmentation. The Haralick texture feature chosen is the entropy of the retinal image.

In order to performing the proper extraction of the enhanced segments from the Gabor filter response images, an effective thresholding method is required.

Assume that a Gabor filter response image has a size of $\mathrm{M}$ * $\mathrm{N}$ with $\mathrm{L}$ grey levels denoted by $\mathrm{G}=\{0,1 \ldots \mathrm{L}-1\}$. A cooccurrence matrix of an image is an $\mathrm{L} * \mathrm{~L}$ square matrix, denoted by $T=\left|t_{i j}\right|_{L \times L}$ whose elements are specified by the numbers of transitions between all pairs of grey levels in $\mathrm{G}=$ $\{0,1 \ldots \mathrm{L}-1\}$ in a particular way.

That gives an idea about the transition of intensity between adjacent pixels, indicating spatial structural information of image. Depending upon the ways in which the gray level $i$ follows gray level $j$, different definition of co-occurrence matrix are possible. Here, we made the co-occurrence matrix asymmetric by considering the horizontally right and vertically lower transitions. Let $t_{i j}$ be the $(i, j)$ th entry of the cooccurrence matrix. Then the probability of co-occurrence $I_{i j}$ of gray levels $i$ and $j$ is Normalizing the probability within individual quadrants, such that the sum of probabilities of each quadrant equals to one, we get the following cell probability. 


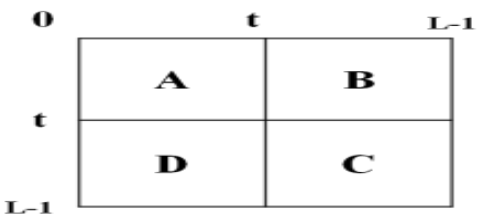

Fig 3: Quadrants of co-occurrence matrix

Let $\mathrm{t}$ be a value used to threshold an image. It partitions the co-occurrence matrix into four quadrants, namely, A, B, C and $\mathrm{D}$. We assume that pixels with grey levels above the threshold are assigned to the foreground (corresponding to objects), and those equal to or below the threshold are assigned to the background. Then quadrants $\mathrm{A}$ and $\mathrm{C}$ correspond to local transitions within background and foreground, respectively, whereas quadrants B and D are joint quadrants which represent joint transitions across boundaries between background and foreground. The probabilities associated with each quadrant are then given by

$$
P_{i j}=\frac{t_{i j}}{\sum_{i} \sum_{j} t_{i j}}
$$

Obviously $0 \leq P_{i j} \leq 1$

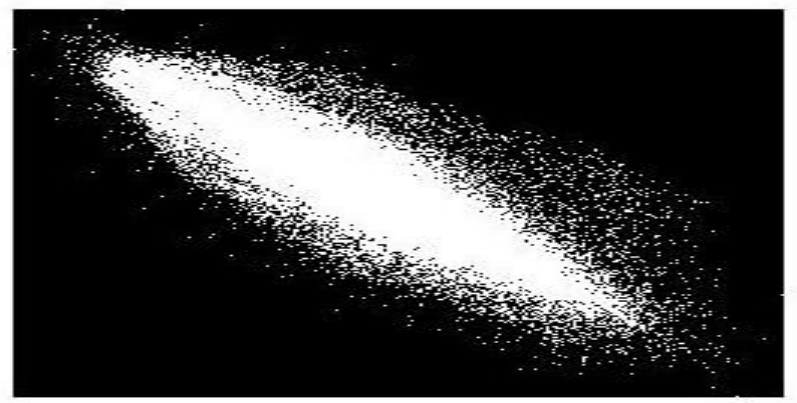

Fig 4: Scatter plot obtained by plotting the local entropy of the optimized Gabor Filter retinal response image

$$
\begin{aligned}
& P_{i j}^{(1)}=\frac{t_{i j}}{\sum_{i=0}^{s} \sum_{j=0}^{s} t_{i j}} \\
& P_{i j}^{(2)}=\frac{t_{i j}}{\sum_{i=s+1}^{L-1} \sum_{j=s+1}^{L-1} t_{i j}}
\end{aligned}
$$

The second order local entropy of the object can be defined as

$$
H^{(A)}(s)=-\frac{1}{2} \sum_{i=0}^{s} \sum_{j=0}^{s} P_{i j}^{(1)} \log _{2} P_{i j}^{(1)}
$$

Similarly the background written as

$$
\begin{aligned}
& H^{(C)}(s)=-\frac{1}{2} \sum_{i=s+1}^{L-1} \sum_{j=s+1}^{L-1} P_{i j}^{(2)} \log _{2} P_{i j}^{(2)} \\
& H_{T}(s)=H^{(A)}(s)+H^{(C)}(s) \\
& t^{*}=\arg \left\{\max H_{T}(s)\right\}
\end{aligned}
$$

The entropy threshold determines the optimal threshold $t^{*}$ by maximum of the entropy curve. $t *$ is used as the threshold for segmentation of the retinal image. This Threshold find it automatically form the Entropy-Threshold Curve.

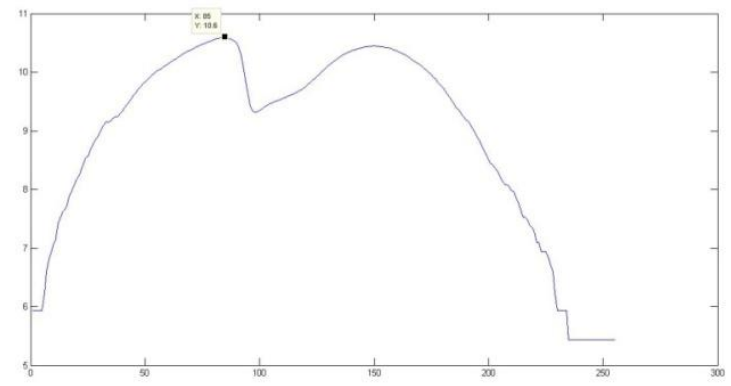

Fig 5: Local entropy curve of the foreground object form quadrants $\mathrm{A}$ and $\mathrm{C}$ of the scatter plot
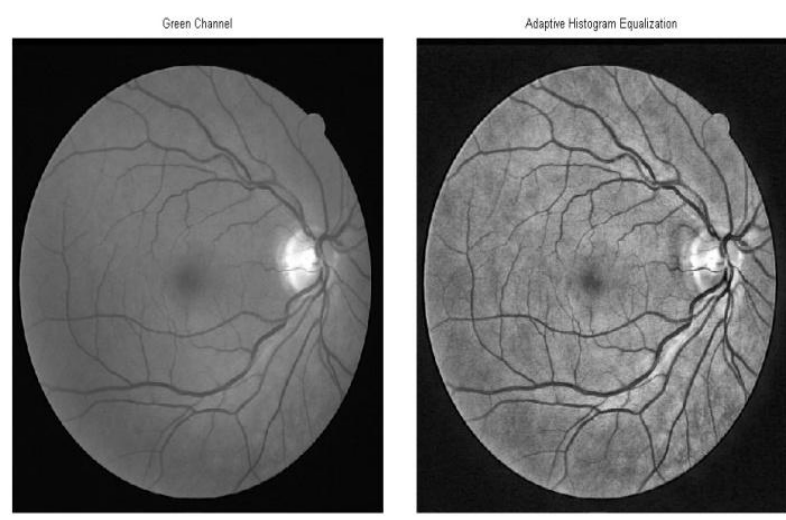

Fig 6: Green channel of the original image (left) and Adaptive histogram equalization image (right)

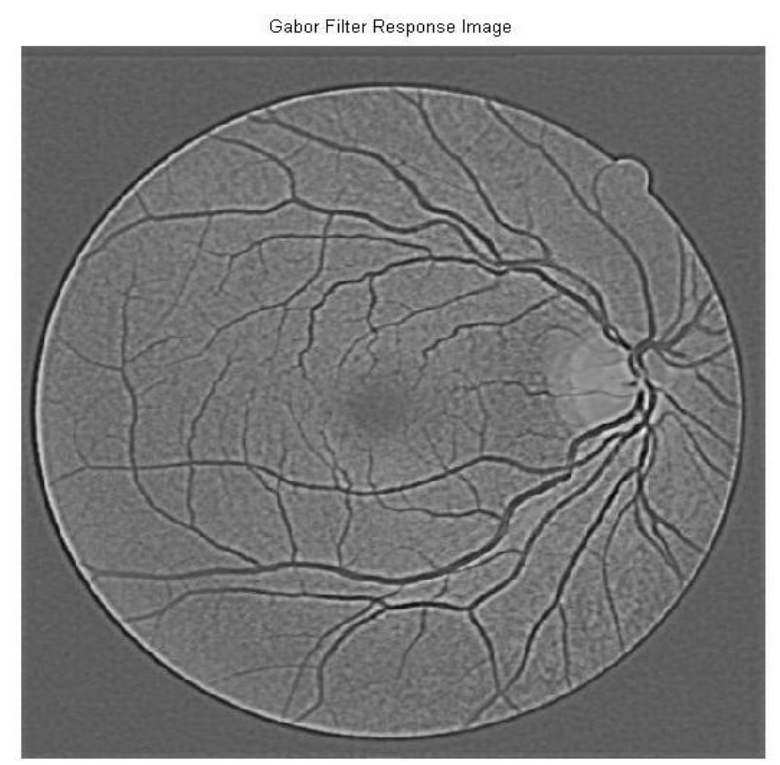

Fig 7: Gabor Filter Response Image

\section{RESULTS AND DISCUSSIONS}

This paper, the software selected to perform the experiment is MATLAB 7.10 (R2010a). The retinal images were collected from the DRIVE database. The accuracy $\left(A_{c c}\right)$ is calculated by the ratio of the number of correctly classified pixels to the total number of pixels in the image. This method average accuracy is $97.94 \%$. The sensitivity $\left(S_{e}\right)$ represents the fraction of pixels correctly classified as vessel pixels, where the false 
positive defines the fraction of pixels erroneously classified as vessel pixels. Average sensitivity is $98.5 \%$. The computational time of whole process of our method takes approximate 2 seconds for each retinal image.

Accuracy $\left(A_{c c}\right)$

$$
A_{c c}=\frac{T P+T N}{T P+T N+F P+F N}
$$

Sensitivity $\left(S_{e}\right)$

$$
S_{e}=\frac{T P}{T P+F N}
$$

Table 1. Vessels Classification

\begin{tabular}{|l|l|c|}
\hline & \multicolumn{1}{|c|}{ Vessel Present } & Vessel Absent \\
\hline Vessel detected & True Positive (TP) & False Positive (FP) \\
Vessel not detected & False Negative (FN) & True Negative (TN) \\
\hline
\end{tabular}

Table 2. Segmentation Results for DRIVE Database

\begin{tabular}{|c|c|}
\hline Image & Accuracy $($ Acc $)$ \\
\hline 1 & 0.9918 \\
\hline 2 & 0.9834 \\
\hline 3 & 0.9839 \\
\hline 4 & 0.9827 \\
\hline 5 & 0.9853 \\
\hline 6 & 0.9774 \\
\hline 7 & 0.9767 \\
\hline 8 & 0.9779 \\
\hline
\end{tabular}
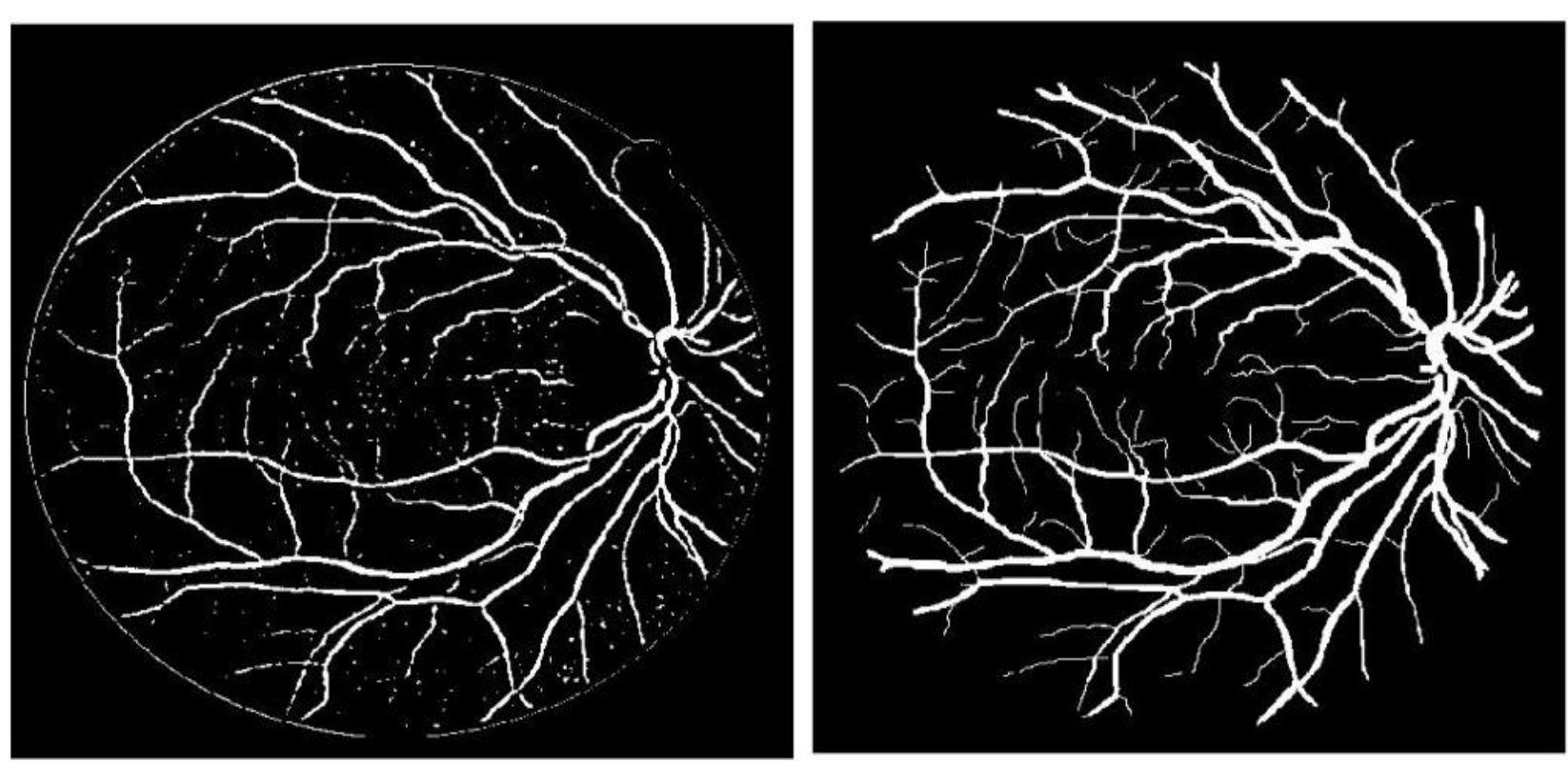

Fig 8: Proposed Segmented Image (left) and Gold Standard Image (right)

\begin{tabular}{|c|c|}
\hline 9 & 0.9726 \\
\hline 10 & 0.9716 \\
\hline 11 & 0.9733 \\
\hline 12 & 0.9758 \\
\hline 13 & 0.9725 \\
\hline 14 & 0.9743 \\
\hline 15 & 0.9729 \\
\hline 16 & 0.9895 \\
\hline 17 & 0.9783 \\
\hline 18 & 0.9789 \\
\hline 19 & 0.9898 \\
\hline 20 & 0.9797 \\
\hline Average Accuracy & $\mathbf{0 . 9 7 9 4}$ \\
\hline
\end{tabular}

Table 3. Performance Comparison with other Methods for DRIVE Database

\begin{tabular}{|c|c|}
\hline Method & Accuracy $\left(\boldsymbol{A}_{c c}\right)$ \\
\hline Chaudhuri et al.[10] & 0.8773 \\
\hline Staal et al.[12] & 0.9441 \\
\hline Jiang and Mojon [13] & 0.8911 \\
\hline Niemeijer et al.[20] & 0.9417 \\
\hline Mendonca et al. [21] & 0.9463 \\
\hline Cinsdikici and Aydin [22] & 0.9293 \\
\hline Proposed method & $\mathbf{0 . 9 7 9 4}$ \\
\hline
\end{tabular}




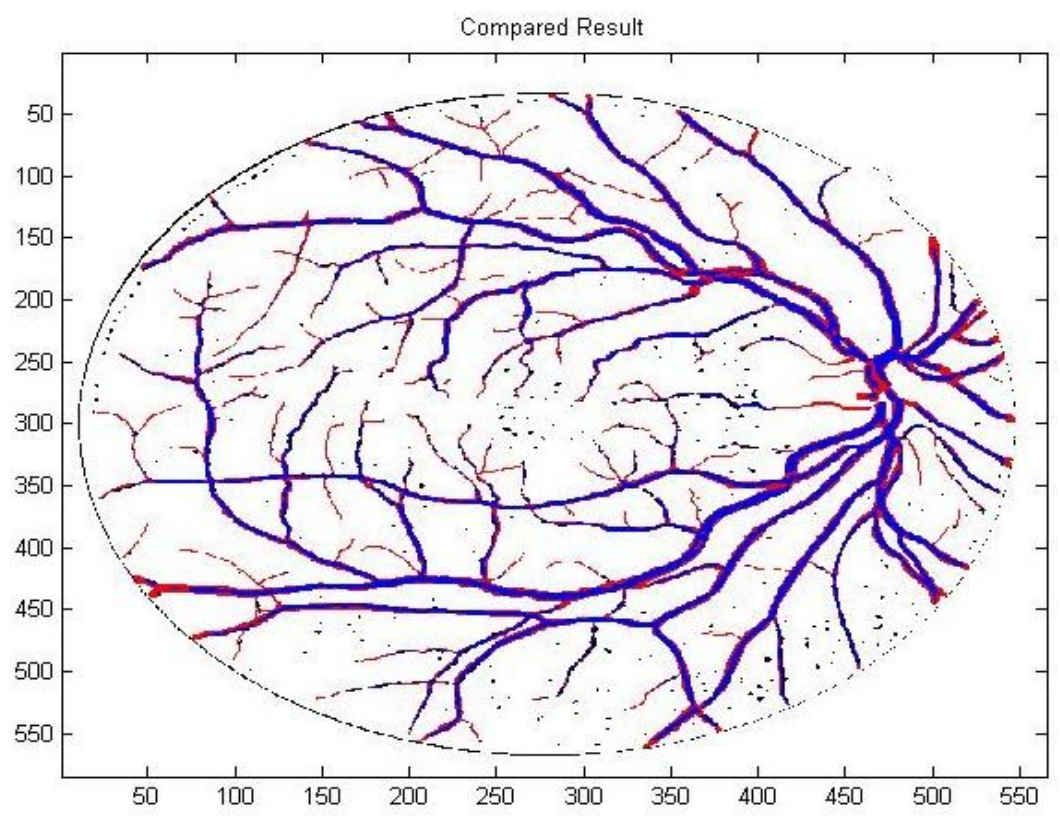

Fig 9: Compared Image (Gold standard \& Final resultant image)

[True Positive $($ TP $)=$ Blue pixels, False Positive $($ FP $)=$ Black pixels,

False Negative $(\mathrm{FN})=$ Red pixels, True Negative $(\mathrm{TN})=$ White pixels $]$

\section{CONCLUSION}

A fully automated retinal blood vessels segmentation method has been presented. In this paper, first introduce optimized Gabor filter with local entropy thresholding for vessels extraction. The threshold value obtained by calculating the entropy feature from the gray level co-occurrence matrix of the optimal Gabor filter retinal image can be one of the ways of segmentation of retinal images. This analysis demonstrated higher true positive rate and reduce false detection in retinal image. The performance of the proposed method is evaluated by comparing DRIVE database images [30]. This method average accuracy $\left(A_{C C}\right)$ is $97.94 \%$ and average sensitivity ( $S e$ ) is $98.5 \%$. The accuracy of the algorithm may be improved by effective noise reducing techniques. The algorithm has less computations and elapsed time is approximate 2 seconds for segmentation of one retinal image. This analysis may also be applied to publically available STARE database. This method can be applied for image registration purpose to track the change in fundus images for monitoring diabetic retinopathy. In this automated segmentation method will help ophthalmologists screen larger populations for blood vessels abnormalities in retinal image.

\section{REFERENCES}

[1] American Diabetes Association. Standards of medical care for patients with diabetes mellitus. Diabetes Care 2000; 23: S32-S42.

[2] Alireza Osareh and Bita Shadgar, "Retinal Vessel Extraction Using Gabor Filters and Support Vector Machines," Advances in Computer Science and Engineering Communications in Computer and Information Science Volume 6, 2009, pp 356-363

[3] Alauddin Bhuiyan, Baikunth Nath, Joselito Chua and Ramamohanarao Kotagiri, "Blood Vessel Segmentation From Color Retinal Images Using Unsupervised Texture Classification," Image Processing, 2007. ICIP 2007.
IEEE International Conference on Vol: 5, Publication Year: 2007, Page(s): V - 521 - V - 524

[4] P.C. Siddalingaswamy, K. Gopalakrishna Prabhu, "Automatic Segmentation of Blood Vessels in Colour Retinal Images using Spatial Gabor Filter and Multiscale Analysis," 13th International Conference on Biomedical Engineering, IFMBE Proceedings Volume 23, 2009, pp 274-276 Springer

[5] Wu, D.; Ming Zhang; Jyh-Charn Liu; Bauman, W., "On the adaptive detection of blood vessels in retinal images," Biomedical Engineering, IEEE Transactions on , vol.53, no.2, pp.341,343, Feb. 2006

[6] Fraz, M.M.; Remagnino, P.; Hoppe, A.; Velastin, S.; Uyyanonvara, B.; Barman, S.A., "A supervised method for retinal blood vessel segmentation using line strength, multiscale Gabor and morphological features," Signal and Image Processing Applications (ICSIPA), 2011 IEEE International Conference on , vol., no., pp.410,415, 16-18 Nov. 2011

[7] D. S. Fong, L. Aiello, T. W. Gardner, G. L. King, G. Blankenship, J. D. Cavallerano, F. L. Ferris, and R. Klein, "Diabetic retinopathy," Diabetes Care, vol. 26, pp. 226-229, 2003.

[8] S. J. Lee, C. A. McCarty, H. R. Taylor, and J. E. Keeffe, "Costs of mobile screening for diabetic retinopathy: A practical framework for rural populations," Aust. J. Rural Health, vol. 8, pp. 186-192, 2001.

[9] American Academy of Ophthalmology Retina Panel, Preferred Practice Pattern Guidelines. Diabetic Retinopathy. San Francisco, CA, Am. Acad. Ophthalmo., 2008 [Online]. Available: http://www.aao.org/ppp.

[10] S. Chaudhauri, S. Chatterjee, N. Katz, M. Nelson and M. Goldbaum, "Detection of blood vessels in retinal images 
using two dimensional matched filters," IEEE Trnas. Medical imaging, vol. 8, no. 3, September 1989.

[11] A. Hoover, V. Kouznetsova, and M. Goldbaum, "Locating blood vessels in retinal images by piecewise threshold probing of a matched filter response," IEEE Trans. Medical imaging, vol. 19, no. 3, March 2000.

[12] J. Staal, M. D. Abràmoff, M. Niemeijer, M. A. Viergever, and B. v. Ginneken, "Ridge based vessel segmentation in color images of the retina," IEEE Trans. Med. Imag., vol. 23, no. 4, pp. 501-509, Apr. 2004.

[13] X. Jiang and D. Mojon, "Adaptive local thresholding by verificationbased multithreshold probing with application to vessel detection in retinal images," IEEE Trans. Pattern Anal. Mach. Intell., vol. 25, no. 1,pp. 131-137, Jan. 2003.

[14] J. V. B. Soares, J. J. G. Leandro, R. M. Cesar, Jr., H. F. Jelinek, and M. J. Cree, "Retinal vessel segmentation using the 2D Gabor wavelet and supervised classification," IEEE Trans. Med. Imag., vol. 25, no. 9, pp. 1214-1222, Sep. 2006.

[15] S. K. Kuri, S. S. Patankar and J. V. Kulkarni "Optimized MFR \& automated local entropy thresholding for retinal blood vessel extraction" Proc. $7^{\text {th }}$ Int. Conf. ICECE, pp. 141- 144, 2012.

[16] Pun, T., 'A new method for grey-level picture thresholding using the entropy of the histogram', Signal Process., 1980, 2, pp. 223-237

[17] Kapur, J.N., Sahoo, P.K., and Wong, A.K.C.: 'A new method for greylevel picture thresholding using the entropy of the histogram', Comput. Vis. Graph. Image Process. 1985, 29, pp. 273-285

[18] C. I. Chang, Y. Du, J. Wang, S.-M. Guo and P.D. Thouin, "Survey and comparative analysis of entropy and relative entropy thresholding techniques," IEE Proc.Vis. Image Signal Process., Vol. 153, No. 6, December 2006

[19] R. M. Haralick, K. Shanmugan, I. Dinstein, "Textural Featues for Images Classification," IEEE Trans. System, Man and Cybernetics.Vol. SMC-3, No - 6, Nov1973, 610-621.

[20] M. Niemeijer, J. Staal, B. v. Ginneken, M. Loog, and M. D. Abramoff, J. Fitzpatrick and M. Sonka, Eds., "Comparative study of retinal vessel segmentation methods on a new publicly available database," in SPIE Med. Imag., 2004, vol. 5370, pp. 648-656.

[21] A. M. Mendonça and A. Campilho, "Segmentation of retinal blood vessels by combining the detection of centerlines and morphological reconstruction," IEEE
Trans. Med. Imag., vol. 25, no. 9, pp. 1200-1213, Sep. 2006.

[22] M. G. Cinsdikici and D. Aydin, "Detection of blood vessels in ophthalmoscope images using MF/ant (matched filter/ant colony) algorithm," Comput. Methods Programs Biomed., vol. 96, pp. 85-95, 2009.

[23] S. J. Lee, C. A. McCarty, H. R. Taylor, and J. E. Keeffe, "Costs of mobile screening for diabetic retinopathy: A practical framework for rural populations," Aust. J. Rural Health, vol. 8, pp. 186-192, 2001.

[24] L. Gang, O. Chutatape, and S. M. Krishnan, "Detection and measurement of retinal vessels in fundus images using amplitude modified second-order Gaussian filter," IEEE Trans. Biomed. Eng., vol. 49, pp. 168-172, Feb. 2002.

[25] H. Li and O. Chutatape, "Automated feature extraction in color retinal images by a model based Approach," IEEE Trans. Biomed. Eng., vol. 51, no. 2, pp. 246-254, Feb. 2004.

[26] M. Foracchia, E. Grisam, and A. Ruggeri, "Detection of the optic disc in retinal images by means of a geometrical model of vessel structure," IEEE Trans. Med. Imag., vol. 23, no. 10, pp. 1189-1195, Oct. 2004.

[27] C. L. Tsai, C. V. Stewart, H. L. Tanenbaum and B. Roysam, "Modelbased method for improving the accuracy and repeatability of estimating vascular bifurcations and crossovers from retinal fundus images," IEEE Trans. Inf. Technol. Biomed., vol. 8, no. 2, pp. 122-130, Jun. 2004.

[28] A. Can, H. Shen, J. N. Turner, H. L. Tanenbaum, and B. Roysam, "Rapid automated tracing and feature extraction from retinal fundus images using direct exploratory algorithms," IEEE Trans. Inf. Technol. Biomed., vol. 3, no. 2, pp. 125-138, Jun. 1999.

[29] J. P. Antoine, P. Carette, R. Murenzi, and B. Piette, "Image analysis with two-dimensional continuous wavelet transform," Signal Process., vol. 31, pp. 241 $272,1993$.

[30] Research Section, Digital Retinal Image for Vessel Extraction (DRIVE) Database. Utrecht, The Netherlands, Univ. Med. Center Utrecht, Image Sci. Inst. [Online]. Available: $\quad$ http://www.isi.uu.nl/Research/Databases/DRIVE

[31] R. C. Gonzalez, R. E. Woods and S. L. Eddins, "Digital Image Processing Using MATLAB," Pearson prentice Hall, 2004

[32] R. Haralick and L. Shapiro, Computer and Robot Vision. vol. 1, Chap. 5, Addision-Wesley, 1992 